\title{
Simple equation of state for hard disks on the hyperbolic plane
}

\author{
Mariano López de Hard* \\ Centro de Investigación en Energía, Universidad Nacional Autónoma de México (U.N.A.M.), Temixco, Morelos 62580, Mexico \\ Andrés Santo@ $†$ and Santos B. Yuste \\ Departamento de Física, Universidad de Extremadura, E-06071 Badajoz, Spain
}

(Dated: October 30, 2018)

\begin{abstract}
A simple equation of state for hard disks on the hyperbolic plane is proposed. It yields the exact second virial coefficient and contains a pole at the highest possible packing. A comparison with another very recent theoretical proposal and simulation data is presented.
\end{abstract}

It is well known that hard-core systems $\frac{1}{1}$ represent useful models that allow both the derivation of some rigorous results in statistical mechanics as well as the computation of some particular quantities such as virial coefficients. For monocomponent hard-core systems there cannot be a gas-liquid transition due to the lack of an attractive part in the intermolecular potential, but they show crystalline and/or amorphous phases and the way these phases arise and even their actual existence are still open problems.

Insight into the thermodynamic behavior of hard-core systems has in the past been sometimes gained by considering similar systems in higher dimensions $\stackrel{2}{2}$ The rationale is that one may obtain a rough idea of the thermodynamic behavior of say three-dimensional hard-core fluids at high density by looking at models in higher dimensions in which the same phenomenology is present but at a lower density and thus the problem may become mathematically more tractable. In a different context, but nevertheless dealing with a somewhat related question, Modes and Kamien ${ }^{\underline{3}}$ have addressed the connection between the equation of state and disordered packings of hard disks. Since in simulations of monodisperse hard disks it is difficult to prevent crystallization, the consideration of a curved surface, in this case a hyperbolic plane with a curvature near a known regular tesselation, serves to frustrate global crystalline order and allows them to find the equation of state via molecular dynamics. Further, they have also developed a free area theory for the packing derived from the nearby tesselation. Studies in the hyperbolic plane in connection with glass-forming liquids and bulk behavior of physical systems have also recently been reported. $\stackrel{4}{=}$

A few years ago we proposed a simple and accurate equation of state for a hard-disk fluid $\underline{\underline{5}}$ This equation is built so as to yield the exact second virial coefficient and also to have a single pole singularity at the close-packing fraction, namely

$$
p / \rho k_{B} T=\left[1-b_{2} \eta-\left(1-b_{2} \eta_{\max }\right) \eta^{2} / \eta_{\max }^{2}\right]^{-1},
$$

where $p$ is the pressure, $\rho$ is the number density, $k_{B}$ is the Boltzmann constant, $T$ is the absolute temperature, $b_{2}=2$ is the reduced second virial coefficient, $\eta=a_{0}(\sigma) \rho$ is the packing fraction, with $a_{0}(\sigma)=(\pi / 4) \sigma^{2}$ the area of a hard disk of diameter $\sigma$, and $\eta_{\max }=\sqrt{3} \pi / 6 \simeq 0.9069$ is the value corresponding to crystalline close-packing.
The major aim of this Note is to provide an answer to the question of whether a proposal such as Eq. (10), properly generalized, may also be useful for hard disks on the hyperbolic plane.

The development goes as follows. First, we note that on a two-dimensional manifold with constant intrinsic curvature $K<0$, the area of a disk of diameter $\sigma$ is $a_{K}(\sigma)=2 \pi|K|^{-1}\left[\cosh \left(|K|^{1 / 2} \sigma / 2\right)-1\right]$ and that the packing fraction is given by $\eta=a_{K}(\sigma) \rho$. Furthermore, on such a manifold the associated (reduced) second virial coefficient depends on the (reduced) diameter $|K|^{1 / 2} \sigma$, namely $b_{2}\left(|K|^{1 / 2} \sigma\right)=a_{K}(2 \sigma) / 2 a_{K}(\sigma) \underline{\underline{3}}$ Thus, for a given value of the reduced diameter $|K|^{1 / 2} \sigma$, the only other requirement in our formulation is the corresponding value of $\eta_{\max }$, irrespective of the fact that the resulting configuration be an ordered one or not. As far as we know, this value is only known analytically for the so-called $\{p, q\}$ tesselations (restricted in the hyperbolic plane by the condition $1 / p+1 / q<1 / 2)$, in which the packing corresponding to the highest possible density and the associated reduced diameter are given by $\eta_{\max }=[\cos (\pi / q) / \sin (\pi / p)-1] /(p / 2-1-p / q)$ and $|K|^{1 / 2} \sigma=2 \cosh ^{-1}[\cos (\pi / q) / \sin (\pi / p)]$, respectively $\underline{\underline{\underline{3}}}$

The top panel of Fig. [1]shows the compressibility factor $Z \equiv p / \rho k_{B} T$ as a function of the packing fraction $\eta$, obtained from the free area theory of Modes and Kamien ${ }^{3}$ and from the use of Eq. (1), in the case of three isostatic tesselations, that is, tesselations with $p=4$. Clearly, the compressibility factors in both approaches are quite close for high packing fractions, but they differ substantially at low ones. We also compare the results for the packing fraction dependence of $Z$ obtained with (to our knowledge) the only presently available simulation data of Modes and Kamien ${ }^{6}$ for two values $\left(|K|^{1 / 2} \sigma=1.062\right.$ and $\left.|K|^{1 / 2} \sigma=1.060\right)$ of the hard-disk diameter which are close to the one corresponding to the isostatic $\{4,5\}$ tesselation, namely $|K|^{1 / 2} \sigma=1.0613$. The performance of both theories with respect to the simulation is rather poor with, if any, a very slight superiority of our approach. Nevertheless, no definite statement about such a comparison should be made at this stage given the very small number of particles (1 to 9) used in the simulations and the intrinsic difficulties associated with simulations on the hyperbolic plane $\underline{\underline{4}}$

To further test the performance of both theories in a 


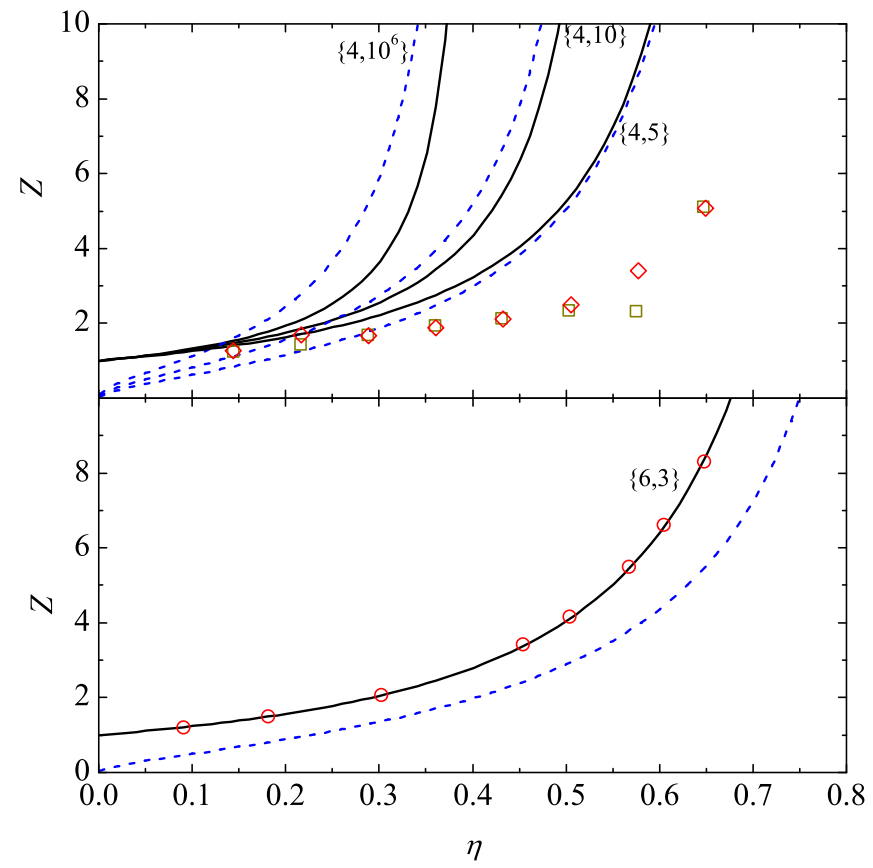

FIG. 1: (Color online) Top panel: Compressibility factor $Z$ as a function of $\eta$ for the isostatic $\left\{4,10^{6}\right\},\{4,10\}$, and $\{4,5\}$ tesselations $\left(|K|^{1 / 2} \sigma=1.7628,1.6169\right.$, and 1.0613, and $\eta_{\max }=0.4142,0.5750$, and 0.7206 , respectively); solid lines: Eq. (1); dashed lines: free area theory of Ref. 3; the symbols are simulation results also obtained in Ref. 3 for $|K|^{1 / 2} \sigma=1.060$ (squares) and $|K|^{1 / 2} \sigma=1.062$ (diamonds). Bottom panel: Compressibility factor $Z$ as a function of $\eta$ for the (Euclidean) hexagonal $\{6,3\}$ tesselation $\left(|K|^{1 / 2} \sigma=0\right.$, $\eta_{\max }=\sqrt{3} \pi / 6$ ); solid line: Eq. (1); dashed line: free area theory of Ref. 3; the symbols are the simulation results from Ref. 7 .

more controllable situation, in the bottom panel of Fig. 1 we present the results obtained in the Euclidean limit $\left(|K|^{1 / 2} \sigma=0\right)$ using the hexagonal $\{6,3\}$ tesselation. Of course in this limit in our approach we recover the results of our earlier proposal for the equation of state of a harddisk fluid, $\underline{\underline{5}}$ which we had proved to be rather accurate. The free area theory,$\underline{\underline{3}}$ on the other hand, performs very poorly.

While the philosophy behind the derivation of our equation of state is totally different from that of the free area theory of Modes and Kamien,,$\underline{3}$ they both share the property of having a pole at $\eta=\eta_{\max }$. In fact, from Eq. (11) it follows that $Z \simeq\left[2-b_{2}\left(|K|^{1 / 2} \sigma\right) \eta_{\max }\right]^{-1}(1-$ $\left.\eta / \eta_{\max }\right)^{-1}$ while the free area theory yields $Z_{\text {free area }} \simeq$ $2\left(1-\eta / \eta_{\max }\right)^{-1}$ for $\eta$ close to $\eta_{\max }$. Note that if
$|K|^{1 / 2} \sigma=1.0613$ then $\left[2-b_{2}\left(|K|^{1 / 2} \sigma\right) \eta_{m}\right]^{-1} \simeq 2.20$. This explains why at high packing fractions the numerical values of the compressibility factor obtained with both approaches are not all that different. One should point out that the free area theory has been constructed with the particular aim of performing well at high densities while ours attempts to capture both the low and high density limits. On the other hand, $Z_{\text {free area }}$ is nonanalytic at $\eta=0$ (for the above value of $|K|^{1 / 2} \sigma, Z_{\text {free area }}=$ $\left.1.262 \eta^{1 / 2}+1.4328 \eta+1.5941 \eta^{3 / 2}+\cdots\right)$ and incorrectly predicts $Z_{\text {free area }}(0)=0$, whereas by construction $Z$ given by Eq. (10) yields the exact second virial coefficient $b_{2}\left(|K|^{1 / 2} \sigma\right)$ and also allows us to estimate the higher order virial coefficients $b_{n}\left(|K|^{1 / 2} \sigma\right)$. Thus, from Eq. (1) and again for $|K|^{1 / 2} \sigma=1.0613$, we get $b_{3}(1.0613)=3.55$, $b_{4}(1.0613)=5.36$, and $b_{5}(1.0613)=7.76$. Comparing with the numerical values obtained by Modes and Kamien ${ }^{6}$ for $|K|^{1 / 2} \sigma=1.10$, namely $b_{3}(1.10)=3.39$, $b_{4}(1.10)=4.62$, and $b_{5}(1.10)=5.83$, we find that our estimates are of the right order of magnitude although their accuracy seems to be worse than the one we got for the Euclidean case.$\underline{\underline{5}}$ As a final comment one should add that Eq. (11) may lead to (unphysical) negative values of $Z$ in the interval $\eta_{\max } /\left[b_{2}\left(|K|^{1 / 2} \sigma\right) \eta_{\max }-1\right]<\eta<\eta_{\max }$ if $b_{2}\left(|K|^{1 / 2} \sigma\right) \eta_{\max }>2$. This may well be a limitation of our formulation but the point certainly deserves further study.

In summary, in this Note we have presented an extension of our former equation of state for a hard-disk fluid to deal with the same system on the hyperbolic plane. In contrast with the free area theory of Ref. 3, which is rather more complicated and devised to perform well in the high density region only, the main assets of our proposal are its simplicity and the fact that it caters both for low and high packing fractions. Our results indicate that it might be as accurate and even improve on the performance of the free area theory of Modes and Kamien $\underline{\underline{3}}$ A deeper assessment of its full value is not possible at this stage due to the lack of simulation results for different values of $|K|^{1 / 2} \sigma$. We hope that our simple theory will encourage the performance of such simulations.

We want to thank C. D. Modes and R. D. Kamien for kindly providing us with their simulation data and their values for the virial coefficients. The work of A.S. and S.B.Y. has been supported by the Ministerio de Educación y Ciencia (Spain) through Grant No. FIS200760977 (partially financed by FEDER funds) and by the Junta de Extremadura through Grant No. GRU08069. M.L.H. acknowledges the partial financial support of DGAPA-UNAM under project IN-109408.

\footnotetext{
* Electronic address: malopez@servidor.unam.mx URL: http://xml.cie.unam.mx/xml/tc/ft/mlh/

$\dagger$ Electronic address: andres@unex.es URL: http://www . unex.es/fisteor/andres/
}

$\ddagger$ Electronic address: santos@unex.es, URL: http://www.unex.es/fisteor/santos/

1 For a very recent review of the properties of these systems one may look at Theory and Simulation of Hard-Sphere Flu- 
ids and Related Systems, edited by A. Mulero (Springer, Berlin, 2008).

2 A few illustrative papers are H. L. Frisch and J. K. Percus, Phys. Rev. A 35, 4696 (1987); M. Baus and J. L. Colot, ibid. 36, 3912 (1987); H. L. Frisch and J. K. Percus, Phys. Rev. E 60, 2942 (1999); G. Parisi and F. Slanina, ibid. 62, 6554 (2000); A. Santos and M. López de Haro, ibid. 72, 010501(R) (2005); F. Zamponi, Phil. Mag. 87, 485 (2007); and R. Rohrmann, M. Robles, M. López de Haro, and A. Santos, J. Chem. Phys. 129, 014510 (2008), where many more references to work on hard-core systems in $D$ dimensions may be found.
3 C. D. Modes and R. D. Kamien, Phys. Rev. Lett. 99, 235701 (2007); Phys. Rev. E. 77, 041125 (2008).

${ }^{4}$ F. Sausset and G. Tarjus, J. Phys. A: Math. Gen. 40, 12873 (2007); preprint arXiv:0805.1475, F. Sausset, G. Tarjus, and P. Viot, preprint arXiv:0805.2819

5 A. Santos, M. López de Haro, and S. B. Yuste, J. Chem. Phys. 103, 4622 (1995); M. López de Haro, A. Santos, and S. B. Yuste, Eur. J. Phys. 19, 281 (1998).

${ }^{6}$ C. Modes and R. D. Kamien, private communication.

7 J. J. Erpenbeck and M. Luban, Phys. Rev. A. 32, 2920 (1985). 